\title{
POLÍTICAS PÚBLICAS EM SAÚDE RELACIONADAS AO DIAGNÓSTICO PRECOCE E RASTREAMENTO DO CÂNCER BUCAL NO BRASIL
}

PUBLIC HEALTH POLICIES RELATED TO ORAL CANCER'S EARLY DIAGNOSIS AND SCREENING IN BRAZIL

POLÍTICAS PÚBLICAS DE SALUd RELACIONADAS CON EL DIAGNÓSTICO Y LA DETECCIÓN PRECOZ DE CÁNCER ORAL EN BRASIL

Fabiana Almeida Miranda ${ }^{1}$

Lavínia Oliveira de Araújo 2

Matheus Ribeiro Melo ${ }^{3}$

Ruth Costa Barbosa ${ }^{4}$

Antônio Prates Caldeira ${ }^{5}$

Fernanda Piana Santos Lima Oliveira 6

Palavras-chave:

Atenção Primária em Saúde; Políticas Públicas de Saúde; Programas de Rastreamento; Neoplasias Bucais.

Keywords:

Primary Health Care; Public Health Policy; Mass Screening; Mouth Neoplasms.

Palabras clave:

Atención Primaria de Salud;

Políticas Públicas de Salud; Tamizaje Masivo; Neoplasias de la Boca.

Submetido: 27/10/2019

Aprovado: 04/12/2019

Autor(a) para Correspondência: Fabiana Almeida Miranda Av. José Alves Miranda, 3 Centro - Mato Verde (MG) 39527-000

E-mail: fabianamiranda793@ gmail.com

\section{RESUMO}

Este estudo teve por objetivo identificar as políticas públicas de diagnóstico precoce e rastreamento do câncer bucal no Brasil. Trata-se de revisão integrativa da literatura realizada nas bases Scientific Electronic Library Online (SCIELO), Literatura Latino-Americana e do Caribe em Ciências da Saúde (LILACS) e Biblioteca Virtual em Saúde (BVS). Realizou-se cruzamento dos seguintes descritores: a) Sistema Único de Saúde (SUS); b) atenção primária em saúde; c) políticas públicas de saúde; d) programas de rastreamento; e) detecção precoce do câncer; e f) câncer bucal. Foram incluídos estudos nos idiomas português e inglês, publicados no período de 2008 a 2018, disponíveis em texto integral e acesso livre e gratuito. Os 12 artigos selecionados possibilitaram a identificação de 5 estratégias de prevenção para deteç̧ão precoce e rastreamento do câncer bucal: a) exame visual; b) levantamento da população de risco; c) autoexame bucal; d) vigilância em saúde; e e) fluxos assistenciais fortes. Constatou-se a necessidade de qualificar os profissionais da saúde para o atendimento, além de aumentar a oferta de serviços odontológicos de qualidade.

\footnotetext{
1. Aluna de Graduação em Medicina no Centro Universitário FIPMoc (UNIFIPMoc). E-mail: fabianamiranda793@ gmail.com ORCID: https://orcid.org/0000-0002-4176-4897

2. Aluna de Graduação em Medicina no UNIFIPMoc. E-mail: laviniaodearaujo@gmail.com ORCID: https://orcid. org/0000-0003-4766-0121

3. Aluno de Graduação em Medicina no UNIFIPMoc. E-mail: matheusribeiromelo10@icloud.com 0RCID: https:// orcid.org/0000-0001-5220-2307

4. Aluna de Graduação em Medicina no UNIFIPMoc. E-mail: ruth.costabarbosa.1@gmail.com ORCID: https:// orcid.org/0000-0002-6595-4484

5. Médico. Doutor em Ciências da Saúde pela Universidade Federal de Minas Gerais (UFMG). Coordenador do Curso de Medicina do UNIFIPMoc. E-mail: antoniopcaldeira@hotmail.com 0RCID: https://orcid.org/0000-00029990-9083

6. Cirurgiã-Dentista. Doutora em Saúde Coletiva pela UFMG. Professora no UNIFIPMoc. E-mail: fernandapiana@ gmail.com ORCID: http://orcid.org/0000-0002-8826-6852
} 


\section{ABSTRACT}

This study aimed to identify public policies for oral cancer's early diagnosis and screening in Brazil. This is an integrative literature review conducted in the databases Scientific Electronic Library Online (SciELO), Latin American and Caribbean Health Sciences Literature (LILACS), and Virtual Health Library (VHL). The following descriptors were crossed with each other: a) Brazilian National Health System (Sistema Único de Saúde [SUS]); b) primary health care; c) public health policy; d) mass screening; e) cancer early detection; and f) oral cancer. We included studies in Portuguese and English, published within the period from 2008 to 2018, available in full text with free and open access. The 12 selected articles made it possible to identify 5 prevention strategies for oral cancer's early detection and screening: a) visual examination; b) at-risk population survey; c) oral selfexamination; d) health surveillance; and e) strong health care delivery. The need to qualify health professionals for providing care was noticed, in addition to increase the supply of good-quality dental services.

\section{RESUMEN}

Este estudio tuvo como objetivo identificar las políticas públicas para el diagnóstico y la detección precoz de cáncer oral en Brasil. Esta es una revisión integradora de literatura realizada en las bases de datos Scientific Electronic Library Online (SciELO), Literatura Latinoamericana y del Caribe en Ciencias de la Salud (LILACS) y Biblioteca Virtual de Salud (BVS). Los siguientes descriptores se cruzaron entre sí: a) Sistema Único de Salud de Brasil (Sistema Único de Saúde [SUS]); b) atención primaria de salud; c) políticas públicas de salud; d) tamizaje masivo; e) detección temprana de cáncer; y f) cáncer oral. Se incluyeron estudios en portugués e inglés, publicados en el período de 2008 a 2018, disponibles en texto completo y acceso libre y gratuito. Los 12 artículos seleccionados permitieron identificar 5 estrategias de prevención para el diagnóstico y la detección precoz de cáncer oral: a) examen visual; b) encuesta de población en riesgo; c) autoexamen oral; d) vigilancia de salud; y e) fuerte suministro de atención. Se constató la necesidad de calificar a profesionales de la salud para brindar atención, además de aumentar la oferta de servicios dentales de buena calidad.

\section{INTRODUÇÃO}

0 câncer bucal compreende as neoplasias malignas que têm como localização primária os lábios, a cavidade oral, as glândulas salivares e a orofaringe ${ }^{1}$. Apresentando altas taxas de morbidade e mortalidade, esse tipo de câncer é considerado um importante problema de saúde pública no Brasil². Estimativas do Instituto Nacional de Câncer (INCA) apontam a incidência de 11.200 casos novos de câncer da cavidade oral em homens e 3.500 em mulheres para cada ano do biênio 2018-2019 no país ${ }^{1}$.

0 desenvolvimento do câncer bucal decorre de diversos fatores, que podem ser físicos, químicos, biológicos ou ambientais. 0 perfil epidemiológico dos indivíduos mais acometidos por essa neoplasia é caracterizado por homens, brancos, na faixa etária entre 50 e 70 anos de idade, trabalhadores expostos ao sol e usuários crônicos de tabaco e/ou álcool (ou a associação de ambas as substâncias) ${ }^{3}$. Infecção pelo vírus do papiloma humano (human papillomavirus [HPV]), alimentação inadequada e má higiene bucal também se relacionam com a carcinogênese da cabeça e do pescoço ${ }^{4}$.

Embora seja o quinto câncer mais frequente em homens no Brasil ${ }^{1}$, o diagnóstico tardio do câncer bucal continua a ser a principal causa de mau prognóstico, demonstrando a necessidade de ações para seu controle, com ênfase em detecção precoce, intervenção nos fatores de risco e tratamento ${ }^{5}$.

Para combater o câncer, políticas públicas de saúde vêm sendo instituídas desde o século XX6. Em 2004, com a publicação das Diretrizes para organização da saúde bucal ${ }^{7}$, no âmbito do Sistema Único de Saúde (SUS), implementou-se o Programa Brasil Sorridente, que, entre outras atividades, compreendia a oferta de serviços voltados ao diagnóstico e à detecção do câncer bucal. A inserção do serviço odontológico nas unidades básicas de saúde (UBS) tem propiciado expansão da cobertura de cuidados em saúde bucal. Entretanto, há necessidade de monitoramento das políticas implementadas para verificar sua efetividade, identificando e corrigindo eventuais falhas ${ }^{8}$.

Nesse contexto, o objetivo deste trabalho foi:

- Identificar as políticas públicas de diagnóstico precoce e rastreamento do câncer bucal no Brasil. 


\section{METODOLOGIA}

Trata-se de revisão integrativa da literatura sobre o câncer bucal, técnica que sistematiza resultados de pesquisa acerca de um assunto específico e, assim, aprofunda o conhecimento sobre o tema investigado por meio de ampla análise de estudos científicos, discutindo métodos e achados de investigação e refletindo com vistas a futuros estudos ${ }^{9}$.

Os artigos foram selecionados nas bases Scientific Electronic Library Online (SciELO), Literatura LatinoAmericana e do Caribe em Ciências da Saúde (LILACS) e Biblioteca Virtual em Saúde (BVS).

No primeiro momento, a estratégia de busca recorreu aos seguintes descritores: Sistema Único de Saúde AND atenção primária em saúde AND políticas públicas de saúde AND programas de rastreamento AND detecção precoce do câncer AND câncer bucal. No segundo momento, manteve-se o descritor que representa o eixo temático e os demais foram cruzados estabelecendo a seguinte estratégia de busca: câncer bucal AND Sistema Único de Saúde, câncer bucal AND atenção primária em saúde, câncer bucal AND políticas públicas de saúde, câncer bucal AND programas de rastreamento, câncer bucal AND detecção precoce do câncer. 0 propósito desse refinamento foi identificar a relação entre o descritor temático principal e os demais investigados. Ambas as estratégias de busca utilizaram os descritores somente em língua portuguesa, considerando que o objetivo do estudo enfatiza políticas públicas desenvolvidas e aplicadas no Brasil.

Os critérios de inclusão adotados foram: a) estudos em português e inglês; b) publicações no período de 2008 a 2018; c) estudos que tratavam de políticas públicas brasileiras (ações públicas) voltadas ao controle do câncer bucal (diagnóstico precoce e rastreamento); d) estudos disponíveis em texto integral; e e) estudos de acesso livre e gratuito. Já os critérios de exclusão foram: a) estudos não relacionados à temática em foco; e b) estudos que não se enquadram no gênero textual artigo científico.

Para a coleta de dados, os estudos foram organizados com base em título, periódico, ano de publicação, região do país, objetivo, principais resultados e conclusão/recomendação, com a finalidade de responder ao objetivo da pesquisa. Na avaliação inicial dos estudos, realizou-se a leitura do título e, posteriormente, do resumo. Participaram da seleção dos artigos 4 pesquisadores do Centro Universitário FIPMoc (UNIFIPMoc), de Montes Claros (MG), em julho e agosto de 2018. Os estudos selecionados foram lidos na íntegra.

\section{RESULTADOS}

As estratégias de busca possibilitaram a identificação de 704 publicações que poderiam ter relação com o tema em foco. 0 Quadro 1 ilustra sua distribuição nas bases consultadas.

Quadro 1 - Distribuição das publicações e seleção dos artigos para revisão integrativa da literatura.

\begin{tabular}{|l|l|c|c|}
\hline Base de dados & Estratégia de busca & Artigos encontrados & Artigos selecionados \\
\hline & $\begin{array}{l}\text { Câncer bucal AND sistema único de } \\
\text { saúde }\end{array}$ & 3 & 1 \\
\cline { 2 - 4 } SciEL0 & $\begin{array}{l}\text { Câncer bucal AND atenção primária } \\
\text { em saúde }\end{array}$ & 11 & 0 \\
\cline { 2 - 4 } & $\begin{array}{l}\text { Câncer bucal AND políticas públicas } \\
\text { de saúde }\end{array}$ & 4 & 1 \\
\hline & $\begin{array}{l}\text { Câncer bucal AND programas de } \\
\text { rastreamento }\end{array}$ & 1 & 0 \\
\hline & $\begin{array}{l}\text { Câncer bucal AND diagnóstico } \\
\text { precoce }\end{array}$ & 14 & 0 \\
\hline
\end{tabular}




\begin{tabular}{|c|c|c|c|}
\hline Base de dados & Estratégia de busca & Artigos encontrados & Artigos selecionados \\
\hline \multirow{5}{*}{ LILACS } & $\begin{array}{l}\text { Câncer bucal AND Sistema Único de } \\
\text { Saúde }\end{array}$ & 15 & 0 \\
\hline & $\begin{array}{l}\text { Câncer bucal AND atenção primária } \\
\text { em saúde }\end{array}$ & 22 & 3 \\
\hline & $\begin{array}{l}\text { Câncer bucal AND políticas públicas } \\
\text { de saúde }\end{array}$ & 18 & 1 \\
\hline & $\begin{array}{l}\text { Câncer bucal AND programas de } \\
\text { rastreamento }\end{array}$ & 12 & 1 \\
\hline & $\begin{array}{l}\text { Câncer bucal AND diagnóstico } \\
\text { precoce }\end{array}$ & 41 & 4 \\
\hline \multirow{5}{*}{ BVS } & $\begin{array}{l}\text { Câncer bucal AND Sistema Único de } \\
\text { Saúde }\end{array}$ & 10 & 1 \\
\hline & $\begin{array}{l}\text { Câncer bucal AND atenção primária } \\
\text { em saúde }\end{array}$ & 66 & 0 \\
\hline & $\begin{array}{l}\text { Câncer bucal AND políticas públicas } \\
\text { de saúde }\end{array}$ & 6 & 0 \\
\hline & $\begin{array}{l}\text { Câncer bucal AND programas de } \\
\text { rastreamento }\end{array}$ & 147 & 0 \\
\hline & $\begin{array}{l}\text { Câncer bucal AND diagnóstico } \\
\text { precoce }\end{array}$ & 334 & 0 \\
\hline \multicolumn{2}{|r|}{ TOTAL } & \multicolumn{2}{|c|}{12} \\
\hline
\end{tabular}

Fonte: Elaborado pelos autores.

Após a leitura dos títulos, 171 publicações foram excluídas por estarem indexadas em mais de uma base e 521 foram excluídas por não possuírem relação direta com o tema em foco. Assim, a amostra final totalizou 12 artigos que atenderam aos critérios de inclusão adotados.

Os artigos incluídos na amostra são apresentados no Quadro 2. 0 periódico que mais publicou sobre o tema foi a revista Ciência \& Saúde Coletiva (4 artigos). As demais revistas apresentaram apenas 1 publicação: a) Applied Cancer Research; b) Revista Panamericana de Salud Pública; c) Cadernos de Saúde Pública; d) Brazilian Dental Journal; e) Brazilian Oral Research;f) Revista Gaúcha de Odontologia; g) Brazilian Journal of Otorhinolaryngology; e h) Arquivos em Odontologia.

Quadro 2 - Relação dos artigos incluídos no estudo.

\begin{tabular}{|c|c|c|c|}
\hline Título & Revista & Ano & $\begin{array}{l}\text { Região } \\
\text { do país }\end{array}$ \\
\hline $\begin{array}{l}\text { Oral cancer, smoking and public health policies in Brazil: } \\
\text { a brief review }{ }^{10}\end{array}$ & Applied Cancer Research & 2011 & Sul \\
\hline $\begin{array}{l}\text { Estratégias e resultados da prevenção do câncer bucal } \\
\text { em idosos de São Paulo, Brasil, } 2001 \text { a } 2009\end{array}$ & $\begin{array}{l}\text { Revista Panamericana de } \\
\text { Salud Pública }\end{array}$ & 2012 & Sudeste \\
\hline $\begin{array}{l}\text { Abordagem do câncer da boca: uma estratégia para os } \\
\text { níveis primário e secundário de atenção em saúde }{ }^{11}\end{array}$ & Cadernos de Saúde Pública & 2012 & Sul \\
\hline $\begin{array}{l}\text { Reorganization of secondary and tertiary health care } \\
\text { levels: impact on the outcomes of oral cancer screening } \\
\text { in the São Paulo State, Brazil } 12\end{array}$ & Brazilian Dental Journal & 2012 & Sudeste \\
\hline $\begin{array}{l}\text { Constructing public oral health policies in Brazil: issues } \\
\text { for reflection }{ }^{13}\end{array}$ & $\begin{array}{l}\text { Brazilian Oral } \\
\text { Research }\end{array}$ & 2012 & Nordeste \\
\hline $\begin{array}{l}\text { Prevenção e detecção do câncer bucal: planejamento } \\
\text { participativo como estratégia para ampliação da } \\
\text { cobertura populacional em idosos }\end{array}$ & Ciência \& Saúde Coletiva & 2013 & Sudeste \\
\hline
\end{tabular}




\begin{tabular}{|c|c|c|c|}
\hline Título & Revista & Ano & $\begin{array}{l}\text { Região } \\
\text { do país }\end{array}$ \\
\hline $\begin{array}{l}\text { Oral cancer in Brazil: a secular history of Public Health } \\
\text { Policies }\end{array}$ & $\begin{array}{l}\text { Revista Gaúcha de } \\
\text { Odontologia }\end{array}$ & 2014 & Nordeste \\
\hline $\begin{array}{l}\text { Oral cancer preventive campaigns: are we reaching the } \\
\text { real target? }{ }^{14}\end{array}$ & $\begin{array}{l}\text { Brazilian Journal of } \\
\text { Otorhinolaryngology }\end{array}$ & 2015 & Sudeste \\
\hline $\begin{array}{l}\text { Prevalence of oral cancer self-examination among elderly } \\
\text { people treated under Brazil's Unified Health System: } \\
\text { household health survey }\end{array}$ & Ciência \& Saúde Coletiva & 2015 & Sudeste \\
\hline $\begin{array}{l}\text { Maior acesso à informação sobre como prevenir o câncer } \\
\text { bucal entre idosos assistidos na atenção primária à saúde }\end{array}$ & Ciência \& Saúde Coletiva & 2015 & Sudeste \\
\hline $\begin{array}{l}\text { Organização dos serviços públicos de saúde bucal para } \\
\text { diagnóstico precoce de desordens com potencial de } \\
\text { malignização do estado do Rio de Janeiro, Brasil }\end{array}$ & Ciência \& Saúde Coletiva & 2016 & Sudeste \\
\hline $\begin{array}{l}\text { Estratégias para resolutividade assertiva da campanha } \\
\text { de diagnóstico e prevenção do câncer bucal }\end{array}$ & Arquivos em Odontologia & 2016 & Sudeste \\
\hline
\end{tabular}

Fonte: Elaborado pelos autores.

Destaca-se o ano de 2012 (4 artigos publicados), seguido por 2016 (3 artigos). Não houve publicação em $2008,2009,2010,2017$ e 2018. Nos demais anos, registra-se apenas 1 publicação. A região Sudeste se destaca (8 artigos publicados), seguida pelas regiões Sul e Nordeste (2 publicações cada). Não foram identificados artigos provenientes das demais regiões do país.

Os artigos incluídos apresentaram coerência quanto a objetivos, principais resultados e conclusões, possibilitando a discussão de suas ideias.

\section{DISCUSSÃO}

Os artigos incluídos neste estudo sugerem a existência de falhas em relação à prevenção primária do câncer bucal, principalmente quanto à dificuldade para estabelecer políticas públicas voltadas aos seus principais fatores de risco (álcool e/ou tabaco, exposição ao sol, alimentação inadequada, infecção por HPV), além de questões relativas aos profissionais da saúde e aos usuários dos serviços envolvidos ${ }^{15-16}$. Dessa forma, o câncer bucal continua sendo um problema de saúde pública, com indicadores epidemiológicos que não apresentaram melhoras nos últimos anos ${ }^{15}$.

Este estudo direcionou seus resultados à prevenção em nível secundário, já que, apesar das lesões iniciais serem facilmente detectáveis, ainda são diagnosticadas tardiamente, o que acaba resultando em tratamentos mutiladores. Assim, evidencia-se a necessidade de adotar ações de rastreamento ${ }^{15,17}$. A literatura consultada apresentou estratégias de prevenção secundária para a detecção precoce e o rastreamento do câncer bucal que podem ser organizadas nas seguintes categorias: a) exame visual; b) levantamento da população de risco; c) autoexame bucal; d) vigilância em saúde; e e) fortalecimento dos fluxos assistenciais.

A discussão apresentada a seguir destaca os aspectos positivos e negativos, levando em conta as políticas e estratégias identificadas nos artigos submetidos à revisão sistemática.

A principal implicação, quanto a exame visual, envolveu a capacitação dos profissionais da saúde, incluindo o cirurgião-dentista $(C D)$, principalmente devido à variação do perfil epidemiológico da patologia. 0s artigos demonstraram a necessidade de educação continuada para a detecção precoce por parte do CD e da equipe de saúde ${ }^{15,18-19}$.

A educação continuada impacta não apenas os conhecimentos, ela também pode modificar o comportamento dos dentistas. 0 controle mais efetivo do câncer de boca e faringe depende da detecção precoce de alterações da mucosa bucal por meio de exames adequados e confirmação por biópsia. Para isso, a educação profissional deve ser enfatizada, assim como a educação pública ${ }^{20}$. Ademais, a educação continuada pode melhorar a qualidade do serviço prestado, pois proporciona atualização do conhecimento após a formação profissional ${ }^{21}$. 
Para alguns autores, o processo de formação e educação continuada para a identificação precoce do câncer bucal não deveria ser exclusivo para o CD. 0s cursos de Medicina deveriam incluir em seu currículo malignidades da região oral e maxilofacial, para que os acadêmicos se capacitem a identificar seus sinais e sintomas 22 .

0 Manual de especialidades em saúde bucal, de $2008^{23}$, foi criado com o objetivo de minimizar os impactos da falta de informações, orientando gestores e dentistas da rede pública de saúde quanto aos agravos e às práticas de prevenção secundária. Além disso, as Portarias do Ministério da Saúde ns. 1.570 e 1.571 , de 2004, e 599, de 2006, instituíram critérios para os centros de especialidades odontológicas (CEOS), incluindo a Estomatologia, com ênfase na prevenção e no diagnóstico precoce como ação prioritária ${ }^{15,18}$.

0 rastreio visual pode ter impacto em indivíduos de alto risco, produzindo significativa mudança de estágio e taxa de sobrevivência. Entretanto, o exame visual apresenta baixa sensibilidade, principalmente em lesões iniciais, e produz altas taxas de falsopositivo ${ }^{24}$.

Em relação à triagem para o câncer bucal, não há evidências que apoiem ou refutem o uso de exame visual como método de rastreamento do câncer bucal na população geral. Uma triagem voltada ao câncer bucal poderia reduzir a mortalidade se enfocasse a população de risco (indivíduos com pouca probabilidade de frequentar consultórios odontológicos com regularidade $)^{15}$.

0 levantamento da população de risco, como possibilidade de detecção precoce, realizado pelos agentes comunitários de saúde (ACS), apareceu como uma estratégia na prevenção secundária. Nas visitas domiciliares, os ACS registrariam duas das principais categorias de risco para o câncer bucal na ficha A do Sistema de Informação da Atenção Básica (SIAB), além de encaminhar o paciente para consulta odontológica. A visita domiciliar poderia, ainda, proporcionar maior participação em campanhas de prevenção, direcionando a população de maior $\operatorname{risco}^{25-26}$.

Embora a literatura consultada não evidencie que o autoexame reduz a taxa de mortalidade do câncer bucal, como parte de um programa de rastreamento populacional, ele é recomendado para minimizar a morbimortalidade da patologia. 0 que se observou foram propostas de políticas públicas de saúde que incluíssem ações educativas

\section{Uma triagem voltada \\ ao câncer bucal \\ poder ia reduzir amor talidade se \\ enfocasse a população de risco...}

voltadas ao autodiagnóstico e autocuidado, além de ações preventivas, de manutenção da saúde, e reabilitadoras. Ainda se mostra baixa a prevalência do diagnóstico precoce realizado por meio do autoexame bucal ${ }^{26-28}$.

0 autoexame bucal constitui estratégia de prevenção de baixo custo e fácil realização, apesar disso, não se trata de prática aplicada rotineiramente, sendo importante para o diagnóstico precoce. 0s CDs não costumam ensinar o autoexame bucal nem - realizam em busca de lesões que possam levar ao desenvolvimento de câncer na prática clínica. 0 motivo pode estar relacionado ao fato de não saberem fazer, por não o considerar necessário ${ }^{29}$.

0 autoexame bucal é visto como uma boa maneira de levar os pacientes a detectarem cânceres assintomáticos em suas fases iniciais. A mídia poderia ser útil na divulgação dessa prática, mas isso não garantiria um efeito significativo, a longo prazo, na consciência do público ${ }^{30}$. Entretanto, não há provas suficientes que apoiem ou refutem seu uso como forma de rastreio ${ }^{31}$.

0 programa de educação sobre o câncer bucal aumentou a consciência sobre a patologia em uma população submetida a estudo. No entanto, houve baixa adesão para participar da triagem clínica e procurar tratamento médico. 0 autoexame bucal poderia ser usado tanto como modalidade de educação em saúde eficaz quanto como ferramenta de detecção precoce, sensibilizando a população de alto risco diante do câncer bucal e de seus fatores de risco, de modo a auxiliar na detecção precoce e na prevenção em países de alta incidência ${ }^{32}$.

Observou-se que grande parte dos estudos analisados obteve sucesso em campanhas de prevenção e diagnóstico precoce do câncer bucal associadas a campanhas de vacinação contra a gripe para idosos. Tal êxito esteve vinculado às parcerias firmadas entre secretarias de saúde, universidades, hospitais públicos e particulares e entidades de 
classe. Além dessas parcerias, a sistematização dos exames e registros e a capacitação dos profissionais envolvidos foram fortemente apontados como indicadores positivos de sucesso, contribuindo para a resolutividade do cuidado voltado a câncer bucal e para a redução do número de casos confirmados. Em contrapartida, houve relato da não inclusão da população de risco para o câncer bucal nessas campanhas. Alguns autores sugerem ampliar a estratégia para outros grupos etários e outras regiões do país ${ }^{17,25,33}$.

Para a redução das taxas de câncer oral, os profissionais devem identificar indivíduos expostos a fatores de risco, bem como orientá-los para a prevenção da patologia ${ }^{16}$.

Os programas nacionais de controle de câncer auxiliam os governos no uso dos recursos disponiveis em benefício de toda a população, por meio de intervenções baseadas em evidências, vigilância em saúde e investigação ${ }^{34}$. 0 modelo de vigilância em saúde para Doenças e Agravos Não Transmissíveis crônicas (DANT) possibilitou implementar um melhor monitoramento da saúde bucal da população brasileira, aumentando a carga de conhecimento sobre fatores de risco e epidemiológicos, aprimorando o direcionamento da prevenção primária e da promoção da saúde, além de auxiliar a construção de um sistema de informação em saúde bucal ${ }^{35}$.

Fora do Brasil, a Assembleia Mundial da Saúde (AMS) aprovou uma resolução sobre saúde bucal em 2007, incentivando os Estados-membros a adotar e implementar políticas e estratégias para a saúde bucal no século XXI, também voltada à prevenção e ao controle do câncer bucal ${ }^{34}$. Nos Estados Unidos da América (EUA), apesar da instituição de programas de rastreio, ainda há dificuldade de adesão por parte da população. Contudo, tais programas foram associados a a aumento da detecção precoce, mas sem melhora do prognóstico de estágios avançados ${ }^{36}$.

Mesmo após o desenvolvimento do SUS, a implantação da Política Nacional da Atenção Básica (PNAB) e a criação da Política Nacional de Saúde Bucal (PNSB), em 2004, que aumentou o acesso dos brasileiros à saúde bucal, ainda existem desafios para que tal acesso seja pleno e que a assistência seja de qualidade. A criação dos CEOs é citada como forte aliada nesse sentido pelos estudos, mas mesmo o fato do diagnóstico oral ser 1 das 5 especialidades previstas, há a ressalva de não exisitr exigência de habilitação específica do profissional que o executa. 0 cadastramento de domicílios pela Estratégia

\section{A insuficiência da rede de atenção em saúde bucal nos casos de câncer foi apontada como um problema.}

Saúde da Família (ESF) também foi apontado como facilitador para a obtenção de informações sobre a prevenção do câncer bucal, a detecção precoce e o encaminhamento de casos suspeitos ${ }^{17,26-27,33}$.

Os CEOs são uma alternativa válida para complementar as necessidades da população em todo o país e sua distribuição deve ser proporcional ao tamanho da população e às necessidades de saúde bucal de cada região ${ }^{37}$.

A insuficiência da rede de atenção em saúde bucal nos casos de câncer foi apontada como um problema. Foram sugeridos o aumento do acesso aos serviços odontológicos de qualidade, a criação de fluxos assistenciais fortes e as ações de promoção da saúde, como a divulgação de informações de prevençã $0^{18,28}$.

Além disso, um planejamento participativo das equipes das UBS para o desenvolvimento de ações, bem como o aumento da cobertura de exames bucais de prevenção e detecção da patologia, foi apontado como indicador de resolutividade ${ }^{19}$. Considerando a crescente incidência do câncer bucal na população com mais de 60 anos, a incorporação de um exame sistematizado nessa população, como parte da rotina de atendimento na ESF, poderia reduzir a incidência do câncer bucal avançado nessa faixa etária ${ }^{38}$.

Os médicos generalistas encaminham pacientes com câncer bucal mais rapidamente, porém, os CDs são melhores na identificação do câncer bucal assintomático, além da detecção durante consultas de rotina. A demora no diagnóstico, por parte dos profissionais, deve-se à não realização de exame clínico completo e à falta de familiaridade com a patologia ${ }^{30}$.

Os atrasos podem ser atribuídos aos profissionais da saúde em casos de erros diagnósticos ou encaminhamentos inadequados ou tardios. Ademais, quando os pacientes são diagnosticados e encaminhados corretamente, pode ocorrer atraso no atendimento terciário por falta de especialistas, laboratórios ou hospitais - tal situação é mais dramática em economias marcadas pela baixa renda ${ }^{26}$. 


\section{CONCLUSÃO}

Esta revisão integrativa registra que, em relação às ações públicas de saúde voltadas ao diagnóstico precoce e ao rastreamento do câncer bucal, a maioria dos artigos apontam a necessidade de formação dos profissionais da saúde para o atendimento, combinada ao aumento da oferta de serviços odontológicos de qualidade.

Nessa área, os estudos devem ser mais abrangentes para proporcionar o desenvolvimento de ações efetivas de promoção da saúde e prevenção de agravos. Assim, minimizam-se os recursos empregados e potencializam-se os resultados de acordo com as metas e os objetivos traçados. Em relação à população, minimizam-se os efeitos deletérios do diagnóstico tardio.

Os sistemas de vigilância em saúde apresentam impacto direto não somente nos carcinomas bucais, mas na saúde bucal da população geral. E, ainda, mostram-se importantes para o planejamento da prevenção primária. Contudo, parece haver desequilíbrio nas ações governamentais, com altos gastos nos tratamentos de estágios avançados e baixos investimentos em ações e pesquisas com foco na prevenção e no controle do câncer bucal.

\section{CONTRIBUIÇÃO DOS AUTORES}

\author{
Fabiana Almeida Miranda, Lavínia Oliveira \\ de Araújo, Matheus Ribeiro Melo e Ruth Costa \\ Barbosa contribuíram com a realização da pesquisa, \\ o delineamento do estudo e a redação do manuscrito.
} Antônio Prates Caldeira contribuiu com a revisão crítica do manuscrito. Fernanda Piana Santos Lima Oliveira contribuiu com o delineamento do estudo e a revisão crítica do manuscrito.

\section{REFERÊNCIAS}

1. Instituto Nacional de Câncer. Estimativa 2018: incidência de câncer no Brasil. Rio de Janeiro: INCA; 2017.

2. Freire JCP, De Oliveira $C R$, Câmara $A 0$, Valença AMG, Santiago BM. Incidência de neoplasias malignas orais nas regiões brasileiras e capitais do Nordeste. Arch Health Invest [serial on the internet]. 2017 [cited 2019 Dec 5];6(11):535-9. Available from: http://www.archhealthinvestigation.com.br/ArcHI/ article/view/2274

3. Domingos PAS, Passalaqua MLC, De Oliveira
ALBM. Câncer bucal: um problema de saúde pública. Rev Odontol Univ Cid São Paulo (Online) [serial on the internet]. 2014 [cited 2019 Dec 5];26(1):4652. Available from: http://publicacoes.unicid. edu.br/index.php/revistadaodontologia/article/ view $/ 285 / 182$

4. Galbiatti ALS, Padovani-Junior JA, Maníglia JV, Rodrigues CDS, Pavarino EC, Goloni-Bertollo EM. Câncer de cabeça e pescoço: causas, prevenção e tratamento. Braz J 0torhinolaryngol [serial on the internet]. 2013 [cited 2019 Dec 5];79(2):23947. Available from: http://www.scielo.br/ scielo.php?script $=$ sci arttext\&pid $=S 1808-$ $86942013000200018 \& \operatorname{lng}=e n \& n r m=i s o \& t \operatorname{lng}=p t$

5. Bandeira CM, De Almeida AA, Carta CFL, Almeida JD, Tango EK. How to improve the early diagnosis of oral cancer? Braz Dent Sci [serial on the internet]. 2017 [cited 2019 Dec 5];20(4):25-31. Available from: http://bds.ict.unesp.br/index.php/cob/article/ view/1439/1190

6. Martins Filho PRS, Santos TS, Da Silva LCF, Piva MR. Oral cancer in Brazil: a secular history of public health policies. Rev Gaúch Odontol [serial on the internet]. 2014 [cited 2019 Dec 5];62(2):159-64. Available from: http://www.scielo.br/scielo.php?script=sci arttext\&pid=S1981-86372014000200159

7. Brasil. Ministério da Saúde. Secretaria de Atenção à Saúde. Departamento de Atenção Básica. Coordenação Nacional de Saúde Bucal. Diretrizes da Política Nacional de Saúde Bucal. Brasília, 2004. Available from: http://189.28.128.100/dab/docs/ publicacoes/geral/diretrizes_da_politica_nacional_ de saude bucal.pdf.

8. Instituto Nacional de Câncer. Monitoramento das ações de controle dos cânceres de lábio e cavidade oral. Rio de Janeiro: INCA; 2016. (Informativo Detecção Precoce n. 3 ).

9. De Souza MT, Da Silva MD, De Carvalho R. Integrative review: what is it? How to do it? Einstein (São Paulo) [serial on the internet]. 2010 [cited 2019 Dec 5];8(1):102-6. Available from: http:// www.scielo.br/scielo.php?script $=$ sci arttext\&pid $=$ S $167945082010000100102 \& \operatorname{lng}=$ en $\& \operatorname{tn} \mathrm{g}=$ en

10. Loyola Edeny AT, França Beatriz HS, Moysés Samuel J, Moysés Simone T, Bisinelli Júlio C. Oral câncer, smoking and public health polices in Brazil: a brief review. Applied Cancer Research. 2011; 31(3):102-6

11. Torres-Pereira Cassius C., Angelim-Dias Aldo, Melo Nilce Santos, Lemos Jr. Celso Augusto, Oliveira Eder Magno Ferreira de. Abordagem do câncer da boca: uma estratégia para os níveis primário e secundário de atenção em saúde. Cad. Saúde Pública. [serial on the internet]. 2012 [cited 2019 Dec 06]; 28(Suppl): s30-s39. Available from: http://www. 
scielo.br/scielo.php?script $=$ sci arttext \&pid=S0102311X2012001300005\&lng $=$ en

12. Almeida Fernanda Campos Sousa de, Cazal Claudia, Pucca Júnior Gilberto Alfredo, Silva Dorival Pedroso da, Frias Antonio Carlos, Araújo Maria Ercilia de. Reorganization of secondary and tertiary health care levels: impact on the outcomes of oral cancer screening in the São Paulo State, Brazil. Braz. Dent. J. [serial on the internet]. 2012 [cited 2019 Dec 06]; 23(3):241-245. Available from: http://www.scielo. br/scielo.php?script $=$ sci arttext\&pid $=S 0103-$ $\underline{64402012000300010 \& \operatorname{lng}=e n}$

13. Soares Catharina Leite Matos. Constructing public oral health policies in Brazil: issues for reflection. Braz. oral res. [serial on the internet]. 2012 [cited 2019 Dec 06]; 26 (spe1):94-102. Available from: http://www.scielo.br/scielo.php?script=sci arttext \&pid $=$ S1806-83242012000700014\&lng $=$ en

14. Nemoto Renato Paladino, Victorino Alana Asciutti, Pessoa Gregory Bittar, Cunha Lais Lourenção Garcia da, Silva José Antonio Rodrigues da, Kanda Jossi Ledo et al. Oral cancer preventive campaigns: are we reaching the real target?. Braz. j. otorhinolaryngol. [serial on the internet]. 2015 [cited 2019 Dec 06]; 81(1): 44-49. Available from: http://www.scielo.

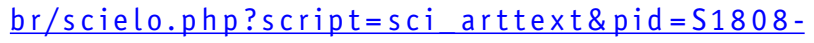
$86942015000100044 \& \operatorname{lng}=$ en

15. Torres-Pereira C. Oral cancer public policies: is there any evidence of impact? Braz Oral Res [serial on the internet]. 2010 [cited 2019 Dec 5];24(Suppl1):37-42. Available from: http://dx.doi. org/10.1590/S1806-83242010000500007

16. De Camargo Cancela M, Voti L, Guerra-Yi M, Chapuis F, Mazuir M, Curado MP. Oral cavity cancer in developed and in developing countries: populationbased incidence. Head Neck [serial on the internet]. 2010 [cited 2019 Dec 5];32(3):357-67. Available from: https://doi.org/10.1002/hed.21193

17. Martins JS, Abreu SCC, Araújo ME, Bourget MMM, Campos FL, Grigoletto MVD, et al. Estratégias e resultados da prevenção do câncer bucal em idosos de São Paulo, Brasil, 2001 a 2009. Rev Panam Salud Pública [serial on the internet]. 2012 [cited 2019 Dec 5];31(3):246-52. Available from: https://www. scielosp.org/pdf/rpsp/2012.v31n3/246-252/pt

18. Casotti E, Monteiro ABF, Castro Filho EL, Santos MP. Organização dos serviços públicos de saúde bucal para diagnóstico precoce de desordens com potencial de malignização do estado do Rio de Janeiro, Brasil. Ciênc Saúde Colet [serial on the internet]. 2016 [cited 2019 Dec 5];21(5):1573-82. Available from: http://dx.doi.org/10.1590/1413-81232015215.10742

19. Bulgareli JV, Diniz OCCF, Faria ET, Vazquez FL, Cortellazzi KL, Pereira AC. Prevenção e detecção do câncer bucal: planejamento participativo como estratégia para ampliação da cobertura populacional em idosos. Ciênc Saúde Colet [serial on the internet]. 2013 [cited 2019 Dec 5];18(12):3461-73. Available from: http://dx.doi.org/10.1590/S1413$\underline{81232013001200003}$

20. Silverman S, Kerr AR, Epstein JB. Oral and pharyngeal cancer control and early detection. J Cancer Educ [serial on the internet]. 2010 [cited 2019 Dec 5];25(3):279-81. Available from: https:// doi.org/10.1007/s13187-010-0045-6

21. Souza JGS, Sá MAB, Popoff DAV. Comportamentos e conhecimentos de cirurgiões-dentistas da atenção primária à saúde quanto ao câncer bucal. Cad Saúde Colet [serial on the internet]. 2016 [cited 2019 Dec 5];24(2):170-7. Available from: http://dx.doi. org/10.1590/1414-462X201600020250

22. Friedrich RE. Delay in diagnosis and referral patterns of 646 patients with oral and maxillofacial cancer: a report from a single institution in Hamburg, Germany. Anticancer Res [serial on the internet]. 2010 [cited 2019 Dec 5];30(5):1833-6. Available from: http://ar.iiarjournals.org/content/30/5/1833. full.pdf

23. Brasil. Ministério da Saúde. Secretaria de Atenção à Saúde. Departamento de Atenção Básica. Manual de especialidades em saúde bucal / Ministério da Saúde, Secretaria de Atenção à Saúde, Departamento de Atenção Básica. - Brasília: Ministério da Saúde, 2008. 128 p.: il. - (Série A. Normas e Manuais Técnicos). Available from: http://bvsms.saude.gov. br/bvs/publicacoes/manual especialidades saude bucal.pdf

24. Brocklehurst $P$, Kujan 0, 0'Malley LA, Ogden G, Shepherd S, Glenny AM. Screening programmes for the early detection and prevention of oral cancer. Cochrane Database Syst Rev (Online) [serial on the internet]. 2013 [cited 2019 Dec 5];(11):CD004150. Available from: https://doi.org/10.1002/14651858. CD004150.pub4

25. Nemoto RP, Victorino AA, Pessoa GB, Cunha LLG, Silva JAR, Kanda Jossi Ledo, et al. Campanha de prevenção do câncer de boca: estamos atingindo o verdadeiro público-alvo? Braz J Otorhinolaryngol [serial on the internet]. 2015 [cited 2019 Dec 5];81(1):44-9. Available from: http://dx.doi.org/10.1016/j.bjorl.2014.03.002

26. Torres-Pereira CC, Angelim-Dias A, Melo NS, Lemos Jr. CA, Oliveira EMF. Abordagem do câncer da boca: uma estratégia para os níveis primário e secundário de atenção em saúde. Cad Saúde Pública [serial on the internet]. 2012 [cited 2019 Dec 5];28(Suppl):s30-s39. Available from: http:// dx.doi.org/10.1590/S0102-311X2012001300005

27. Martins AMEBL, Barreto SM, Santos-Neto PE, Sá 
MAB, Souza JGS, Haikal DS, et al. Maior acesso à informação sobre como prevenir o câncer bucal entre idosos assistidos na atenção primária à saúde. Ciênc Saúde Colet [serial on the internet]. 2015 [cited 2019 Dec 5];20(7):2239-53. Available from: http://www.scielo.br/scielo.php?pid=S1413$\underline{81232015000702239 \& \mathrm{script}=\mathrm{sci} \text { abstract \&tlng}=p t}$

28. Martins AMEBL, Souza JGS, Haikal DS, Paula AMB, Ferreira EF, Pordeus IA. Prevalence of oral cancer self-examination among elderly people treated under Brazil's Unified Health System: household health survey. Ciênc Saúde Colet [serial on the internet]. 2015 [cited 2019 Dec 5];20(4):1085-98. Available from: http://dx.doi.org/10.1590/141381232015204.00542014

29. De Brito Souza LR, Ferraz KD, Pereira NS, Martins MV. Conhecimento acerca do câncer bucal e atitudes frente à sua etiologia e prevenção em um grupo de horticultores de Teresina (PI). Rev Bras Cancerol (Online) [serial on the internet]. 2012 [cited 2019 Dec 5];58(1):31-9. Available from: http://www1.inca. gov.br/rbc/n 58/v01/pdf/06 artigo conhecimento acerca cancer bucal atitudes frente etiologia prevencao grupo horticultores teresina.pdf

30. Gómez I, Warnakulasuriya S, Varela-Centelles PI, López-Jornet P, Suárez-Cunqueiro M, Diz-Dios P, et al. Is early diagnosis of oral cancer a feasible objective? Who is to blame for diagnostic delay? Oral Dis [serial on the internet]. 2010 [cited 2019 Dec 5];16(4):333-42. Available from: https://doi. org/10.1111/j.1601-0825.2009.01642.x

31. Steele T0, Meyers A. Early detection of premalignant lesions and oral cancer. Otolaryngol Clin [serial on the internet]. 2011 [cited 2019 Dec 5];44(1):221-9. Available from: https://doi. org/10.1016/j.otc.2010.10.002

32. Elango KJ, Anandkrishnan N, Suresh A, Iyer SK, RamaIyer SK, Kuriakose MA. Mouth self-examination to improve oral cancer awareness and early detection in a high-risk population. Oral 0ncol [serial on the internet]. 2011 [cited 2019 Dec 5];47(7):6204. Available from: https://doi.org/10.1016/j. oraloncology.2011.05.001

33. Voi PLD, Restini CBA, AL Suzie, Faria Júnior M. Estratégias para resolutividade assertiva da campanha de diagnóstico e prevenção do câncer bucal. Arq 0 dontol [serial on the internet]. 2016 [cited 2019 Dec 5];52(4):221-30. Available from: file:///D:/3720Texto\%20do\%20artigo-12373-1-10-20170818.pdf

34. Petersen PE. Oral cancer prevention and control: the approach of the World Health Organization. Oral Oncol [serial on the internet]. 2009 [cited 2019 Dec 5];45(4-5):454-60. Available from: https://doi. org/10.1016/j.oraloncology.2008.05.023

35. Goes PSA, Figueiredo N, Pucca Júnior GA, Moura L.

Vigilância à saúde bucal: a construção de um modelo integrado. Cad Saúde Pública [serial on the internet]. 2012 [cited 2019 Dec 5];28:S6-S7. Available from: https://www.scielosp.org/scielo.php?pid=S0102$311 \times 2012001300002 \&$ script $=$ sci arttext

36. Downer MC, Moles DR, Palmer S, Speight PM. A systematic review of measures of effectiveness in screening for oral cancer and precancer. Oral Oncol [serial on the internet]. 2006 [cited 2019 Dec 5];42(6):551-60. Available from: https://doi. org/10.1016/j.oraloncology.2005.08.006

37. Pedrazzi V, Dias KRHC, Rode SDM. Oral health in Brazil - Part II: dental specialty centers (CEOs). Braz Oral Res [serial on the internet]. 2008 [cited 2019 Dec 5];22 (Suppl 1):18-23. Available from: http:// www.scielo.br/scielo.php?script $=$ sci arttext\&pid $=\mathrm{S} 1806-83242008000500004$

38. Teixeira EH, Soares CHA, Tajra FS, Mendes Junior FIR, Pereira RS, Aguiar ASW. Sistema de Informação em Saúde Bucal: proposta prermanente de avaliação das ações e serviços de saúde desenvolvidos no Município de Sobral-CE. Sanare (Sobral, Online) [serial on the internet]. 2010 [cited 2019 Dec 5];9(1):8-10. Available from: https://sanare. emnuvens.com.br/sanare/article/view/196
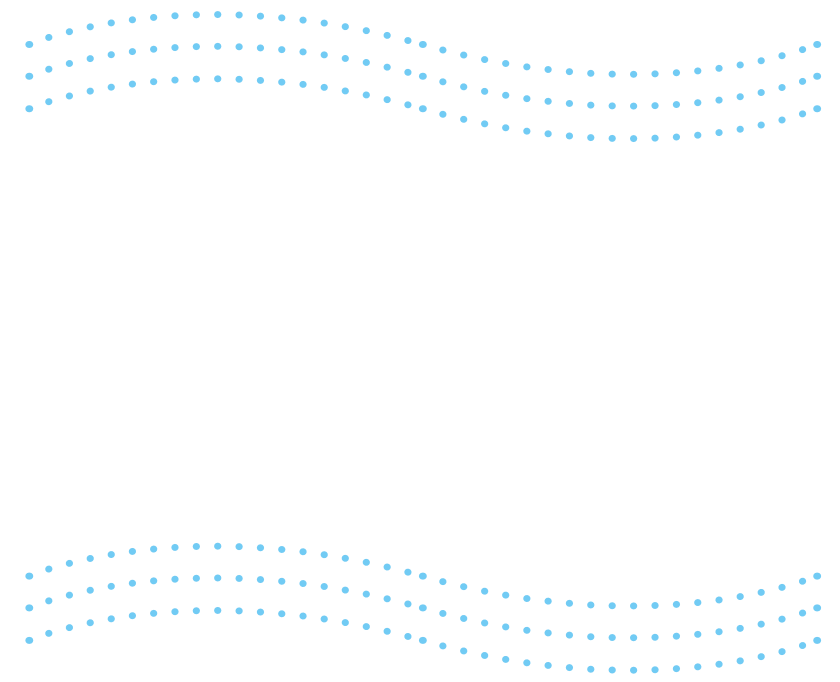
. 